\title{
Evaluation of Micro Fluidic High Pressure Dispersion/Emulsification for SLN Production
}

\author{
J. H. FINKE ${ }^{1}$, J. SCHUR ${ }^{1}$, T. GOTHSCH ${ }^{2}$, S. BEINERT ${ }^{2}$, C. LESCHE ${ }^{3}$, \\ S. BÜTTGENBACH ${ }^{3}$, A. KWADE ${ }^{2}$, C. C. MÜLLER-GOYMANN ${ }^{1}$ \\ ${ }^{1}$ Institut für Pharmazeutische Technologie, Technische Universität Braunschweig, Braunschweig, Germany \\ ${ }^{2}$ Institut für Partikeltechnik, Technische Universität Braunschweig, Braunschweig, Germany \\ ${ }^{3}$ Institut für Mikrotechnik, Technische Universität Braunschweig, Braunschweig, Germany \\ E-mails: jan.finke@tu-bs.de (J. H. Finke), c.mueller-goymann@tu-bs.de (C. C. Müller-Goymann)
}

Sci Pharm. 2010; 78: 587

doi:10.3797/scipharm.cespt.8.LPPT07

To establish a micro fluidic system that covers the whole production process of drug-loaded solid lipid nanoparticles especially high pressure emulsification and dispersion processes must be taken into account. The advantages of micro fluidic systems in a continuous production imply a high surface-to-volume ratio (material and energy transfer) and a narrow residence time distribution (homogeneously defined stresses). Detailed investigation is needed for process understanding and to improve micro channel geometries.

The micro fluidic geometries were designed to selectively expose product flows to different main break-up mechanisms (shear stress, elongational stress, turbulent stress, particle-particle and particle-wall impacts). Within these groups structural modifications were carried out to evaluate their influence on emulsification and dispersion efficiency.

Two formulations (to simulate the dispersion and emulsification process, respectively) were exposed to the specified micro channel geometries (including channel height variation) at different pressures, optionally using multiple homogenisation cycles in addition.

The emulsification process in general shows a decrease in particle size by elevated pressure and higher number of cycles, as expected. Regarding the pressure drop, straight or bent channel geometries show a much lower breakup efficiency compared to either orifice-like or Y-shaped geometries. The latter two types over all show comparable efficiencies. Applying minor structural modifications to several geometries results in significant differences in particle size. As an example, a cascading quadruple orifice geometry shows significantly smaller particle sizes when the flow first interacts with the smallest orifice in comparison to passing the smallest orifice at the end.

The dispersion of solid particles (hydrophilic alumina Alu C) shows similar results although the drop in particle size is less pronounced in these experiments. This is due to the high binding forces between primary particles.

The authors gratefully acknowledge the DFG for financial support of the DFG research group 856 "Mikrosysteme für partikuläre Life-Science-Produkte" (mikroriari). 